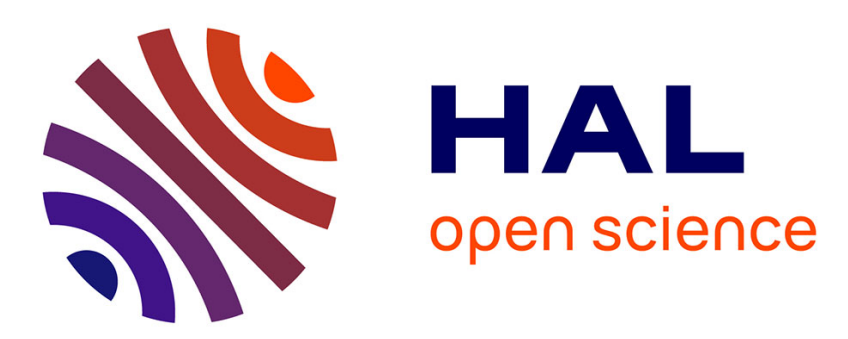

\title{
San Antonio Carupo (centro-norte de Michoacán, México): nuevas evidencias de ciertas transformaciones en el inicio del Postclásico
}

Brigitte Faugère

\section{- To cite this version:}

Brigitte Faugère. San Antonio Carupo (centro-norte de Michoacán, México): nuevas evidencias de ciertas transformaciones en el inicio del Postclásico. Journal de la Société des américanistes, 1991, 77, pp.45-61. 10.3406/jsa.1991.1372 . hal-02909307

\section{HAL Id: hal-02909307 https://hal.science/hal-02909307}

Submitted on 8 Sep 2020

HAL is a multi-disciplinary open access archive for the deposit and dissemination of scientific research documents, whether they are published or not. The documents may come from teaching and research institutions in France or abroad, or from public or private research centers.
L'archive ouverte pluridisciplinaire HAL, est destinée au dépôt et à la diffusion de documents scientifiques de niveau recherche, publiés ou non, émanant des établissements d'enseignement et de recherche français ou étrangers, des laboratoires publics ou privés. 


\section{San Antonio Carupo (centro-norte de Michoacán, México) : nuevas} evidencias de ciertas transformaciones en el inicio del Postclásico Brigitte Faugère-Kalfon

\section{Citer ce document / Cite this document :}

Faugère-Kalfon Brigitte. San Antonio Carupo (centro-norte de Michoacán, México) : nuevas evidencias de ciertas transformaciones en el inicio del Postclásico. In: Journal de la Société des Américanistes. Tome 77, 1991. pp. 45-61; doi : https://doi.org/10.3406/jsa.1991.1372

https://www.persee.fr/doc/jsa_0037-9174_1991_num_77_1_1372

Fichier pdf généré le 05/05/2018 


\title{
Resumen
}

Un análisis de la organization interna y de la morfologia de los edificios del sitio de San Antonio Carupo, ubicado sobre la vertiente meridional del río Lerma (Michoacán, México) permite estudiar las transformaciones que se dieron localmente al inicio del Postclásico (900-1200 DC). En el marco más amplio de la evolution del poblamiento de las regiones centro-occidentales de Mexico en esta época, la interpretación de tales transformaciones da lugar a nuevas hipótesis sobre la extensión de las migraciones proto-toltecas y sobre la génesis del pueblo tarasco.

\section{Résumé}

San Antonio Carupo (Centre-Nord du Michoacán, Mexique) : nouvelles évidences de certaines transformations au début du Postclassique. Une analyse de l'organisation interne et de l'architecture du site de San Antonio Carupo, situé sur le versant méridional du Lerma au Michoacán, Mexique, permet ici d'étudier les transformations qui eurent lieu à cet endroit au début du Postclassique (9001200 ap. J.C.). Replacée dans le cadre plus large de l'évolution du peuplement des régions centreoccidentales du Mexique à cette époque, l'interprétation de ces transformations débouche sur de nouvelles hypothèses concernant tant l'extension des migrations proto-toltèques que la genèse du peuple tarasque.

\begin{abstract}
San Antonio Carupo (North-center of Michoacán, Mexico) : new evidences of some transformations at the beginning of the Postclassic. An analysis focused on the internal organization and architecture of the San Antonio Carupo site, localized on the southern slope of Lerma River in Michoacán, Mexico, leads to a study of the local transformations at the beginning of the Postclassic (900-1200 AD). Replaced on the wider scale of the evolution of settlements in Western-Central regions of Mexico at this time, the interpretation of these transformations leads to new hypotheses concerning the extension of the proto-Toltec migrations as well as the genesis of the Tarascan people.
\end{abstract}




\title{
SAN ANTONIO CARUPO \\ (CENTRO-NORTE DE MICHOACÁN, MEXICO) : NUEVAS EVIDENCIAS DE CIERTAS TRANSFORMACIONES EN EL INICIO DEL POSTCLASICO
}

\author{
Brigitte FAUGÈRE-KALFON *
}

\begin{abstract}
Un análisis de la organización interna y de la morfología de los edificios del sitio de San Antonio Carupo, ubicado sobre la vertiente meridional del río Lerma (Michoacán, México) permite estudiar las transformaciones que se dieron localmente al inicio del Postclásico (900-1200 DC). En el marco más amplio de la evolución del poblamiento de las regiones centro-occidentales de México en esta época, la interpretación de tales transformaciones da lugar a nuevas hipótesis sobre la extensión de las migraciones proto-toltecas y sobre la génesis del pueblo tarasco.
\end{abstract}

San Antonio Carupo (Centre-Nord du Michoacán, Mexique) : nouvelles évidences de certaines
transformations au début du Postclassique.

Une analyse de l'organisation interne et de l'architecture du site de San Antonio Carupo, situé sur le versant méridional du Lerma au Michoacán, Mexique, permet ici d'étudier les transformations qui eurent lieu à cet endroit au début du Postclassique (900-1200 ap. J.C.). Replacée dans le cadre plus large de l'évolution du peuplement des régions centreoccidentales du Mexique à cette époque, l'interprétation de ces transformations débouche sur de nouvelles hypothèses concernant tant l'extension des migrations proto-toltèques que la genèse du peuple tarasque.

San Antonio Carupo (North-center of Michoacan, Mexico) : new evidences of some transformations at the beginning of the Postclassic.

An analysis focused on the internal organization and architecture of the San Antonio Carupo site, localized on the southern slope of Lerma River in Michoacan, Mexico, leads to a study of the local transformations at the beginning of the Postclassic (900-1200 AD). Replaced on the wider scale of the evolution of settlements in Western-Central regions of Mexico at this time, the interpretation of these transformations leads to new hypotheses concerning the extension of the proto-Toltec migrations as well as the genesis of the Tarascan people.

* Proyecto Michoacán, CEMCA, Mexique

J.S.A. 1991, LXXVII : p. 45 à 61. 
En los años recientes, se han registrado algunos avances no despreciables en la comprensión de las transformaciones demográficas, sociales, políticas e ideológicas que se dieron a principios del Postclásico (o, inclusive, a partir de la caída de Teotihuacán) en el centro-occidente de México. Parte de los nuevos conocimientos ha sido conseguida en la cuenca de México misma y en áreas próximas a ella, en particular en Tula (R.H. Cobean y A.G. Mastache, 1989). Pero un buen número de datos $\mathrm{y}$, por lo tanto, de ideas novedosas, es el resultado de varios estudios consagrados a zonas y sitios localizados en la llamada «zona marginal » de la frontera septentrional de Mesoamérica. Respecto a este sector, la multiplicación de los trabajos realizados en los Estados de Michoacán, Guanajuato y Zacatecas (C. Castañeda et alii, 1988, 1989, M.A. Hers, 1989, D. Michelet et alii, 1989, C. Trombold, 1990, entre otros) proporciona una serie de informaciones interesantes que permiten plantear sobre nuevas bases el problema general del reacomodo cultural que parece caracterizar el inicio del período postclásico.

Las investigaciones efectuadas en el norte de Michoacán por el Centro de Estudios Mexicanos y Centro-Americanos (CEMCA) entre 1983 y 1987 figuran entre estos estudios. Si la zona de trabajo, ubicada al sur del río Lerma (Figura 1), ha revelado muchos detalles acerca de su historia prehispánica que no conciernen el momento clave del cual nos ocupamos aquí, existen, en la sub-región de la vertiente meridional del Lerma, diferentes indicios de las transformaciones del Postclásico. Por un lado, es allí donde se sitúa, en el Postclásico Tardío, la frontera entre tarascos y chichimecas (B. Faugère-Kalfon, en preparación). Pero el estudio diacrónico de los asentamientos locales, a partir de la segunda parte del Clásico, nos proporciona varias enseñanzas en cuanto a los cambios en la ocupación humana poco antes del surgimiento de la entidad política tarasca. Entre otros datos que tienen relevancia para nuestro tema, la morfología arquitectónica del sitio de Los Palacios de San Antonio Carupo (MICH. 103) parece indicar la llegada al sur del Lerma (o el impacto de su influencia) de grupos foráneos belicosos, en fechas tan tempranas como el principio del Postclásico Temprano. Movimientos migratorios han sido reportados en Jalisco-Zacatecas (M.A. Hers, 1985 y 1989) y en Guanajuato (B. Braniff, 1988, C. Castañeda et alii, 1988), pero en sentido contrario, es decir que estas regiones fueron, al parecer, en gran parte desocupadas entre 800 y 1000 de nuestra era. Se quiere proponer aquí la hipótesis según la cual las poblaciones "proto-toltecas» (B. Braniff, $1988: 62$ ) del norte de Jalisco y suroeste de Zacatecas también se movieron directamente al sur, hasta el sur de Guanajuato y el norte de Michoacán, para formar un núcleo «proto-tarasco » y que los elementos étnico-culturales que permitieron la constitución del señorío tarasco $y$, por consecuencia, de la frontera norte-central de la Mesoamérica protohistórica deben buscarse mucho antes del siglo XIV.

\section{SAN ANTONIO CARUPO, MICHOACAN : PRESENTACION}

El pueblo actual de San Antonio Carupo se ubica en una zona de transición ecológica, llamada por Rzedowzky (1987) « Bajíos de Michoacán », y comprendida 


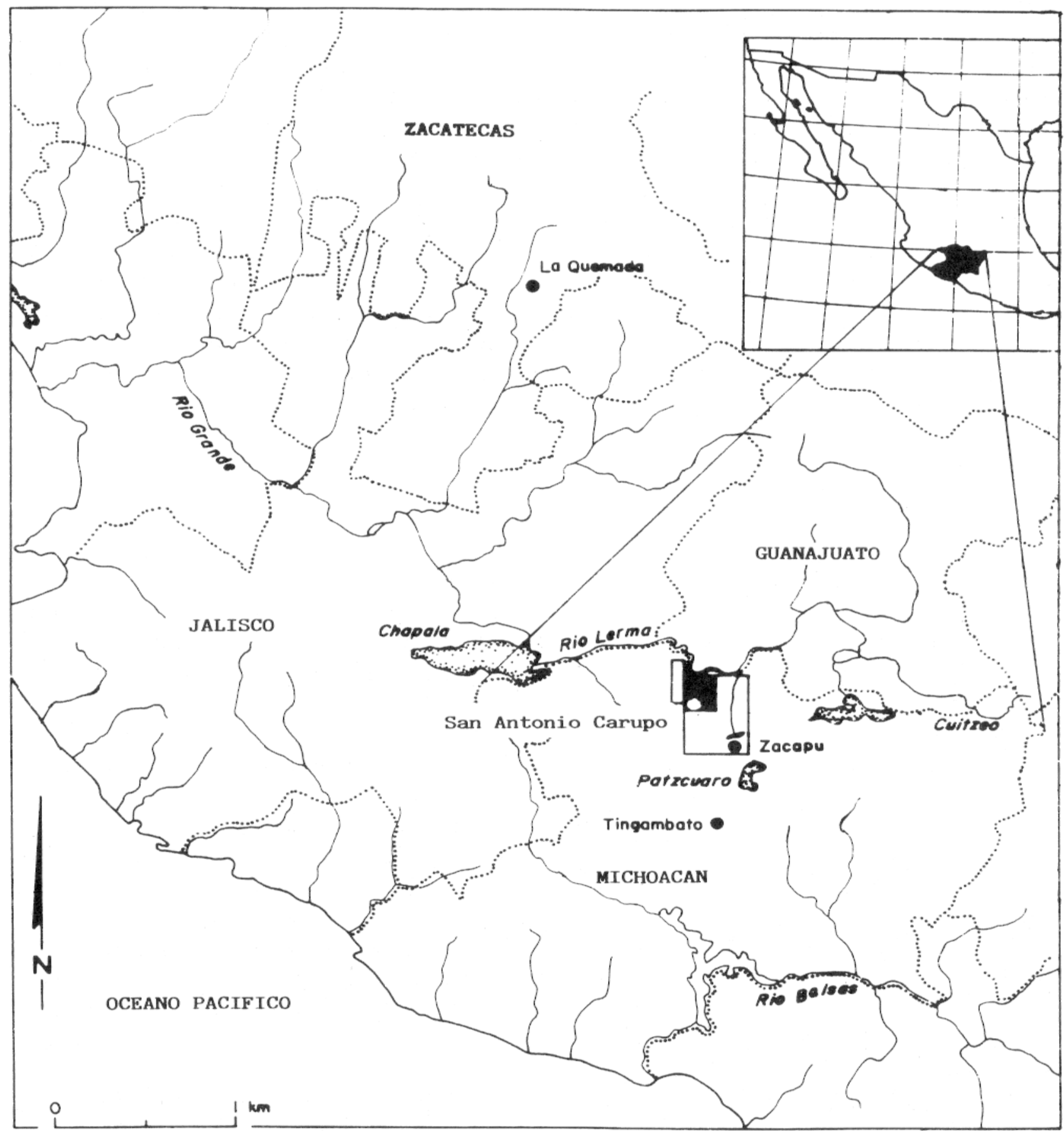

ZONA DEL PROYECTO MICHOACAN DEL CEMCA

SECTOR DE LA "VERTIENTE LERMA".

Fig. 1. - Mapa del centro-occidente de México con la localización de San Antonio Carupo. 
entre las tierras altas de la meseta tarasca al sur, y el bajío guanajuatense. Esta región presenta una pendiente que baja hacia el norte, pero incluye algunas pequeñas depresiones rodeadas de cerros volcánicos bastante elevados. El sitio prehispánico, llamado localmente «El Palacio », está localizado sobre la ladera norte de uno de estos cerros, arriba del pueblo actual (Municipio de Penjamillo).

Se conocía la existencia de este sitio mucho antes del comienzo de los trabajos del CEMCA en Michoacán. Ya en 1904, C. Lumholtz, después de haber descrito las « yácatas » y las fortificaciones del sitio vecino a Zacapu, señalaba su presencia en estos términos : "Análogos monumentos antiguos se pueden encontrar en un espacio de treinta millas al norte de Zacapu, hasta San Antonio Corupo (« quemado en superficie ») ». A finales de los años 70, el Instituto Nacional de Antropología e Historia realizó allí trabajos de despejo, consolidación y restauración de las principales estructuras arquitectónicas (R. Carrasco, 1982). Sin embargo, los conocimientos de orden general, fuera de los datos específicamente arquitectónicos, quedaban limitados, ya que ni el problema de la cronología, ni el del contexto cultural había sido acometido.

\section{EL SITIO}

«El Palacio de San Antonio Carupo » se ubica a 19\%59'32" de latitud norte y $101^{\circ} 54^{\prime} 28^{\prime \prime}$ de longitud oeste, a una altura media de 2.100 metros, en la vertiente norte del Cerro El Agostadero ${ }^{1}$. Representa uno de los sitios más extendidos de la sub-región, con una superficie que se aproxima al kilómetro cuadrado pero, a la vez, es muy poco nucleado : se caracteriza por el hecho de tener varios grupos de estructuras situados más o menos a la misma altura sobre lomillos contiguos. Se encuentran así, de este a oeste, los lomillos « Los Bolaños », « El Cristero », «Alto », « Mocho », «El Palacio » y « Los Llanitos ».

Sobre el lomillo «Los Bolaños » se puede apreciar una pequeña red de terrazas agrícolas y una cueva en forma de pozo de más o menos 8 metros de profundidar Sobre «El Cristero » se encuentran algunas terrazas y cimientos de casas. En «. lomillo «Alto » existen : una estructura rectangular de 15 por 10 metros construída sobre una plataforma baja, una estructura redonda de 3 metros de diámetro y una cámara subterránea $(2,3 \times 3,8$ metros) elaborada en lajas y que tiene un corredor de acceso lateral, hoy en día parcialmente bloqueado, de 3 metros de largo aproximadamente. El lomillo « Mocho » comprende dos construcciones cuadradas muy destruídas, de 15 y 20 metros de lado.

El lomillo «El Palacio» comporta el grupo más importante (Figura 2), compuesto de 12 estructuras principales claramente visibles, construídas sobre espacios planos obtenidos por varios nivelamientos de la pendiente ; «El Palacio » propiamente dicho, ubicado en la parte sureste, domina el conjunto. Esta construcción sub-cuadrada ( $28 \times 27,5$ metros) posee muros de 2,5 metros de ancho, conservados hasta 3 metros de alto, y cinco pilares rectangulares agrupados alrededor de un pequeño patio de 10 metros de lado. Frente a la fachada (oeste), se encuentra un pórtico de 3 metros de ancho delimitado por 3 pilares rectangulares e interrumpido por el ángulo sureste de la cancha de juego de pelota. Este espacio da a una plaza rectangular, originalmente enlosada, dando acceso, al sur, a una alta 


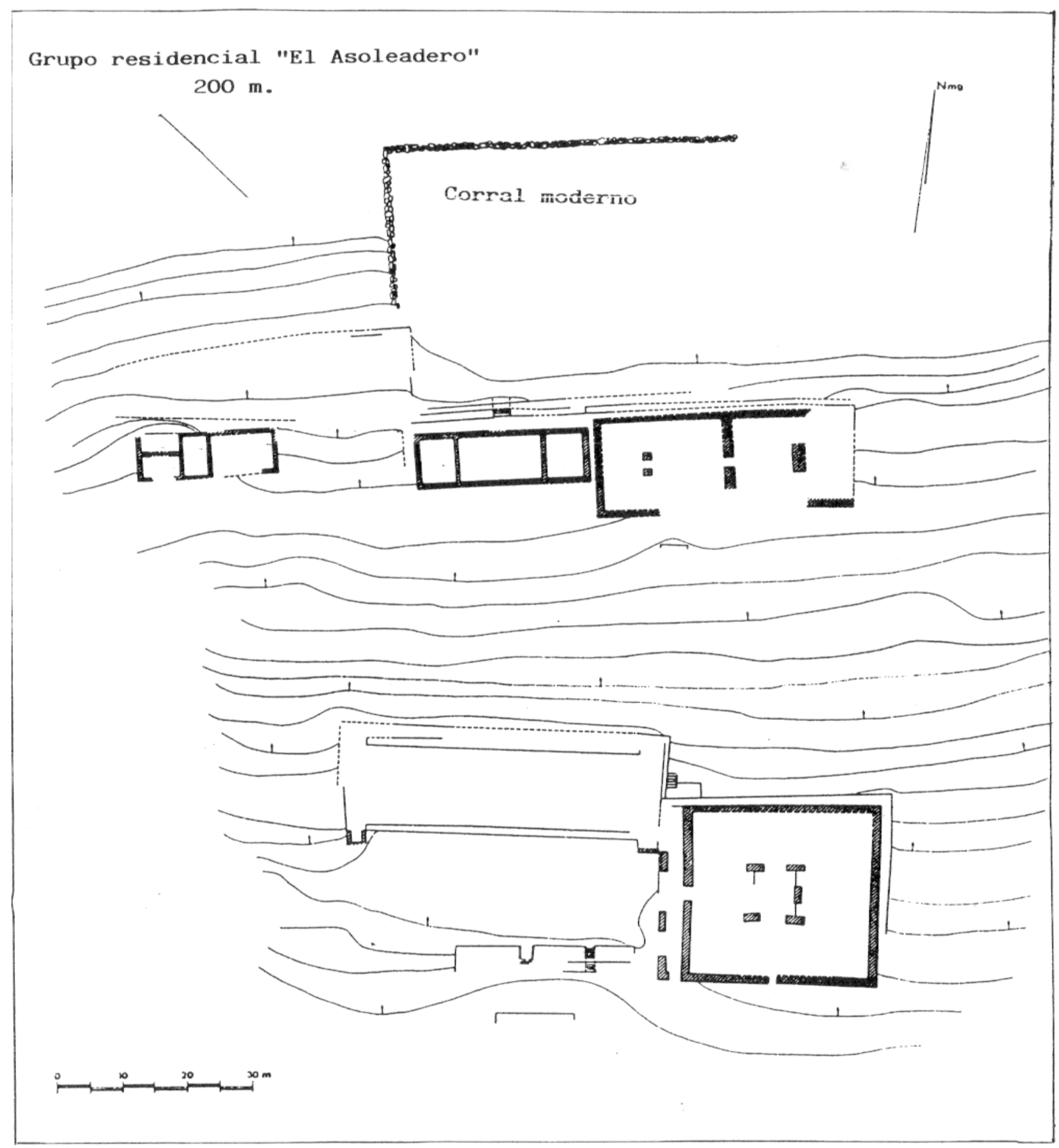

Fig. 2. - Plano del grupo principal del sitio «El Palacio de San Antonio Carupo » (Mich. 103) 
plataforma con dos escaleras paralelas. Al norte de la plaza, hacia abajo, se ubica la cancha de juego de pelota $(48 \times 16,5 \mathrm{~m}$.) que se caracteriza por sus áreas terminales cerradas y banquetas internas estrechas que rematan en un muro casi vertical. Más abajo todavía, se encuentra un grupo de recintos alineados, sin puertas, de forma rectangular, a veces divididos en cuartos simétricos, y que habrían contenido altares o pilares rectangulares. Algunos montículos de lajas rodean este conjunto y parecen corresponder a vestigios de habitaciones. Sin embargo, las zonas localizadas al este-noreste y al sur del «Palacio » comportan también estructuras importantes que quedan todavía por explorar.

$\mathrm{Al}$ oeste de este grupo principal, sobre el lomillo «Los Llanitos » se localizan, por último, los vestigios de cuatro construcciones dispuestas alrededor de una plaza rectangular.

En la superficie del sitio en general, el material arqueológico es relativemente escaso y los tiestos muchas veces muy erosionados. Solamente el material encontrado en profundidad permitió determinar las épocas de ocupación del sitio.

\section{LA CRONOLOGÍA}

La ubicación de nuestro material dentro de la secuencia cerámica regional, así como algunos fechados ${ }^{14} \mathrm{C}$, nos llevan a pensar que la parte central del sitio ha sido ocupada principalmente, pero no exclusivamente, durante el Postclásico Temprano, entre 900 y 1200 de nuestra era (Figura 3).

La ocupación inicial parece, en realidad, anterior : de hecho, no obstante el mal estado general de los tiestos encontrados en las terrazas del grupo del «Palacio », no faltan entre ellos los monocromos incisos con el motivo de la greca rectangular del tipo «Lupe Inciso », muy característico de la segunda parte del Clásico (D. Michelet et alii, 1989). Algunos otros elementos aislados, como un fragmento de cuchara de mango aplanado y un artefacto rectangular soportando una figurilla, parecido a los pseudo-sellos encontrados en Tingambato (R. Piña Chan y K. Oi, 1982 : 68), son materiales relacionados con la época clásica tardía. El sitio fue, entonces, probablemente habitado durante períodos anteriores al año 900, en el final del Clásico y/o en el Epiclásico, aun si las estructuras actualmente visibles son posteriores. Las excavaciones practicadas en la parcela « El Asoleadero » (Figura 4), localizada en la parte inferior del lomillo principal, nos permitieron lograr una mejor apreciación del material cerámico del sitio. Los tiestos pertenecen, en su mayoría, a tipos monocromos color rojo o café, de pastas finas/medianas y con superficies pulidas; así, se nota la frecuencia relativemente elevada de los tipos «Palacio pulido", variedad Lerma, y «Carupo rojo " con o sin nervadura y negativo (Figura 5, c y a). Numerosos fragmentos pertenecen también al grupo «Hornos », con motivos geométricos pintados en rojo o una decoración plástica (Figura $5, \mathrm{f}$ ) : los motivos pintados, ondulantes y paralelos, se parecen mucho a los que se encuentran en la cerámica « Mazapa rojo sobre café », variedad Mazapa de la fase Corral Terminal de Tula (900-950). En realidad, en lo que toca a la cerámica, es la proporción substancial de este grupo que permitió inicialmente colocar la estructura El Asoleadero en la fase Palacio (900-1200 DC). 


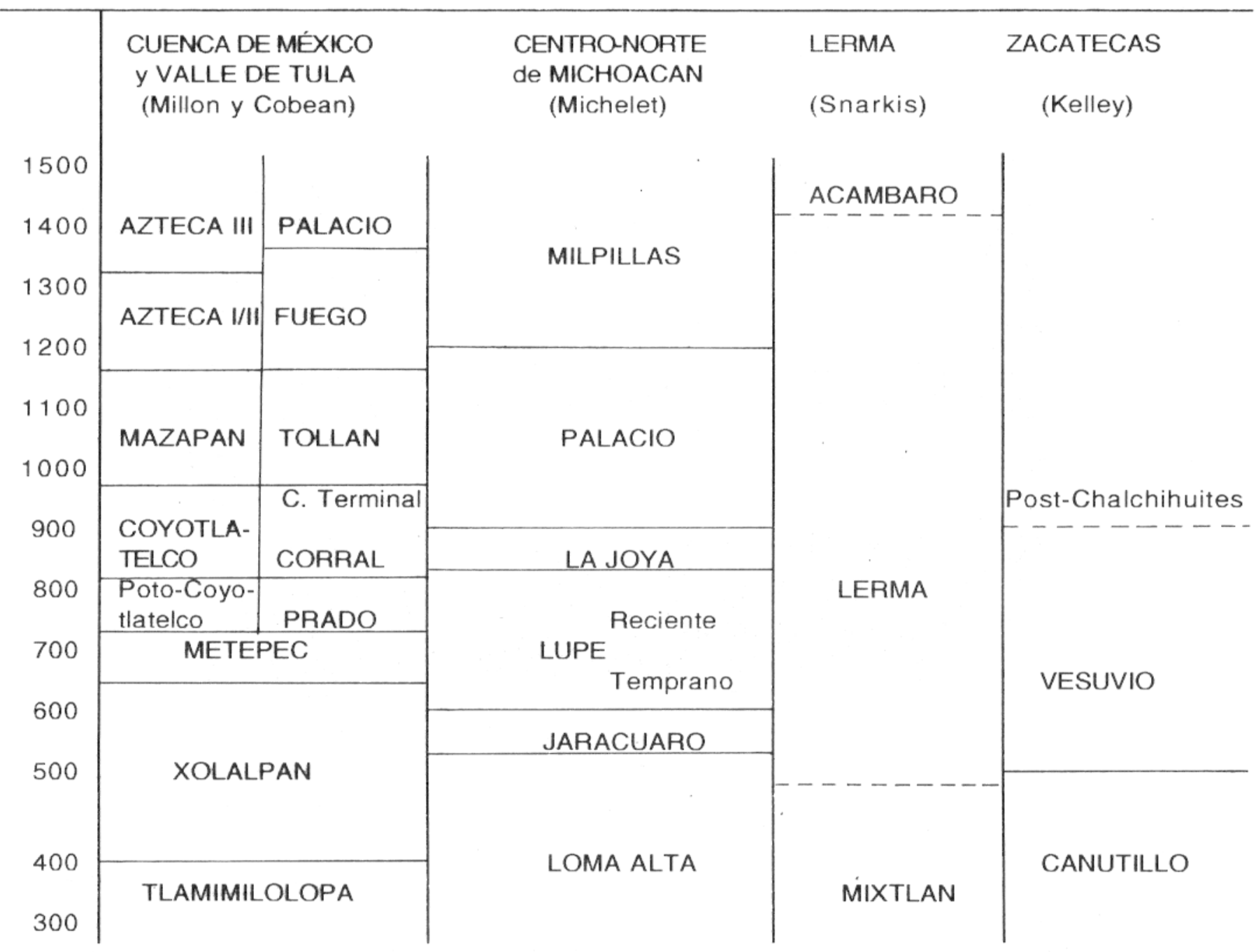

Fig. 3. - Cuadro cronológico.

Casi todos los tipos cerámicos encontrados en San Antonio Carupo existen en los complejos definidos más al sur de la sub-región y cuya evolución, desde el Preclásico Superior, se puede entender en una perspectiva regional (D. Michelet, en preparación). No obstante, queda claro que existen algunas variaciones locales que dan a nuestra colección una cierta originalidad.

La ubicación cronológica de la estructura « El Asoleadero » ha sido confirmada por un fechado ${ }^{14} \mathrm{C}$ proveniente del nivel del piso de la casa (cuadros A y Bsur, capa 3 ; INAH $713: 1100+/-110 \mathrm{BP}$, o sea entre 910 y $975 \mathrm{DC}$ en fechas calibradas). 


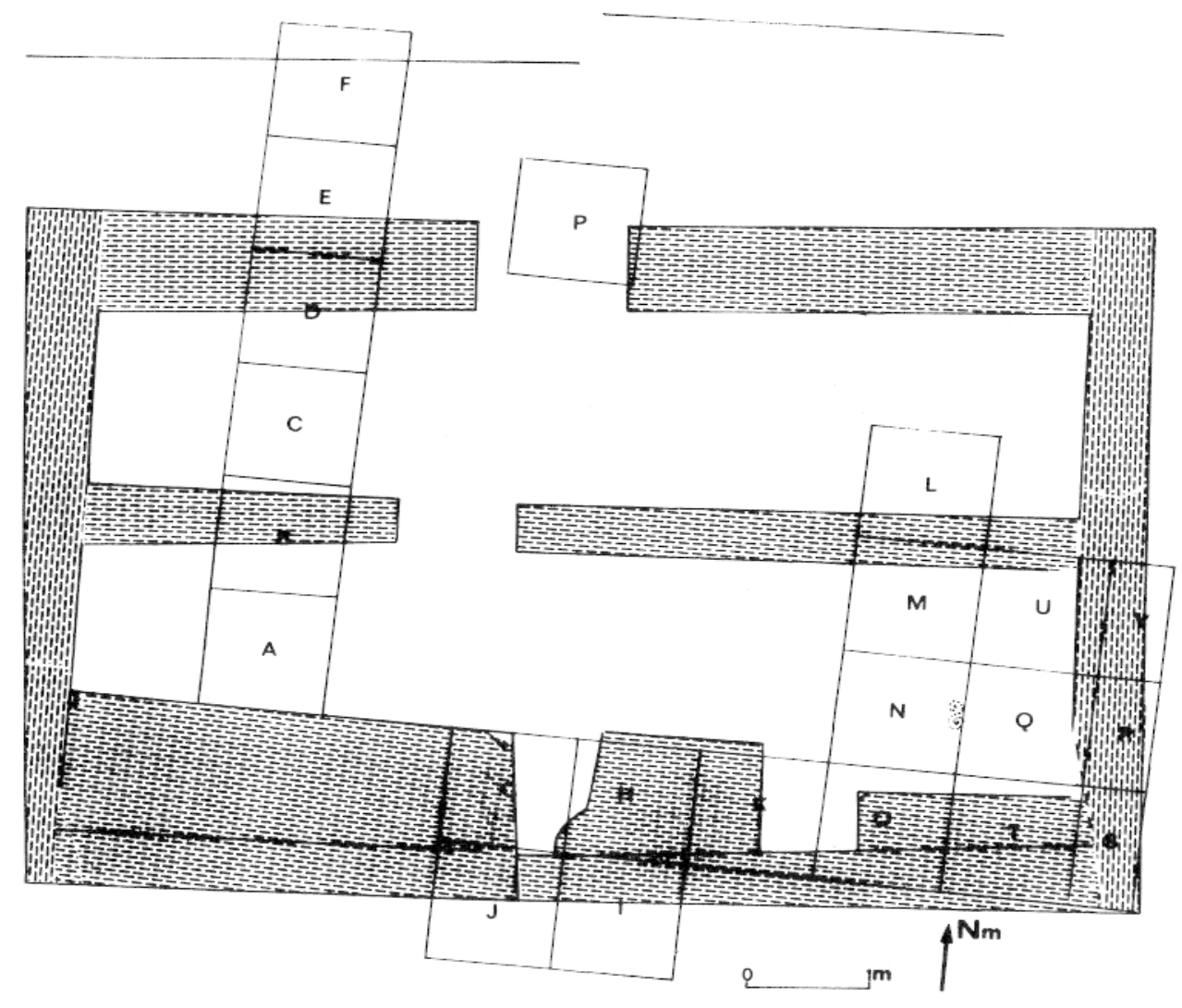

FIG. 4. - Plano de la estructura «El Asoleadero », con las unidades de excavación.

La identidad de las técnicas de construcción entre esta estructura y los edificios públicos del grupo central hace pensar que todo ha sido planificado y realizado al mismo tiempo y pertenece, sin duda, a una sola fase cronológica. Los muros están hechos con lajas de basalto bastante delgadas, siendo algunas de ellas talladas; ningún mortero las une, pero todas parecen dispuestas con mucho cuidado. Una excelente cohesión de las paredes ha sido obtenida por la inserción de pequeños fragmentos triangulares sirviendo de calces.

En El Asoleadero, una ocupación de época tarasca pudo también ser identificada; en el ángulo sureste hemos encontrado dos sepulturas de niños de 10-12 años con mutilaciones dentarias del tipo A2 de la clasificación de Romero, muy común entre los tarascos. Un cascabel de cobre, o de bronce, estaba asociado a uno de los entierros (sepultura 2). Por otra parte, un fechado ${ }^{14} \mathrm{C}$ podría confirmar una intervención tardía sobre la estructura (INAH 714 bis, cuadro L, capa $4: 480+/-60 \mathrm{BP}$, o sea entre 1409 y 1444 DC después de su calibración). Aparentemente, estos elementos del Postclásico Reciente evocan más bien una intervención esporádica y sólo los sectores norte y este de la estructura habrían sido reocupados o simplemente reutilizados para depósitos funerarios. La hipótesis de 


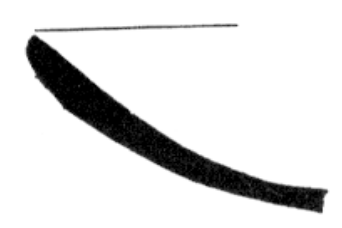

2.
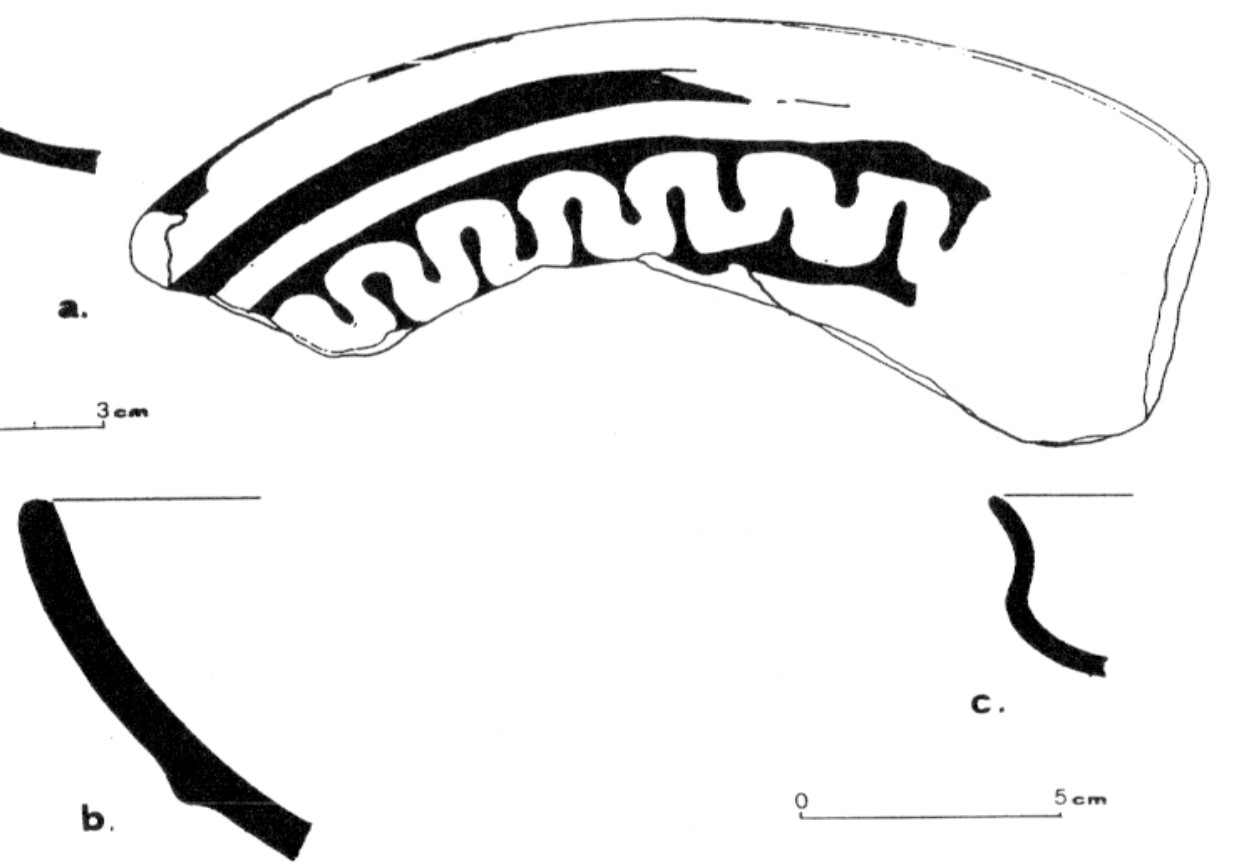

C.

b.
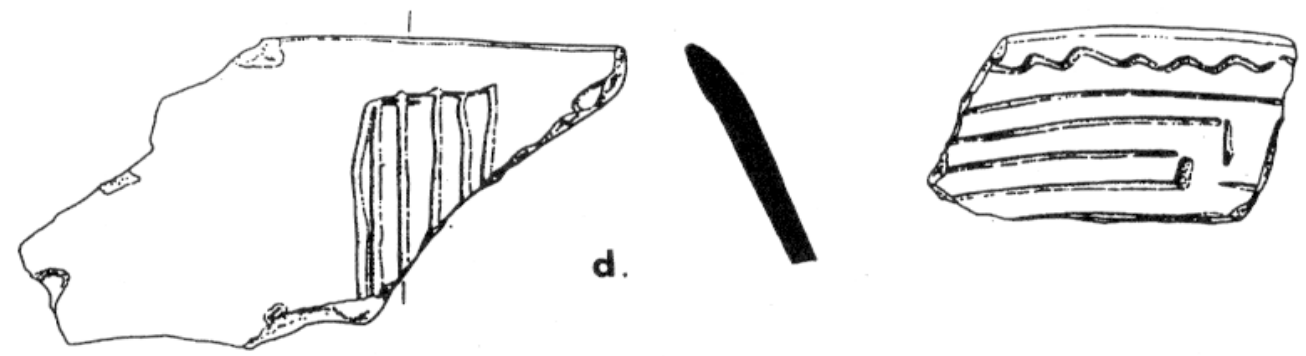

e.

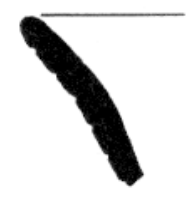

d.
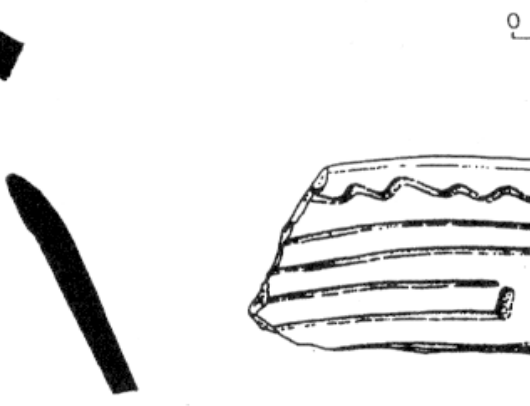
$5 \mathrm{~cm}$
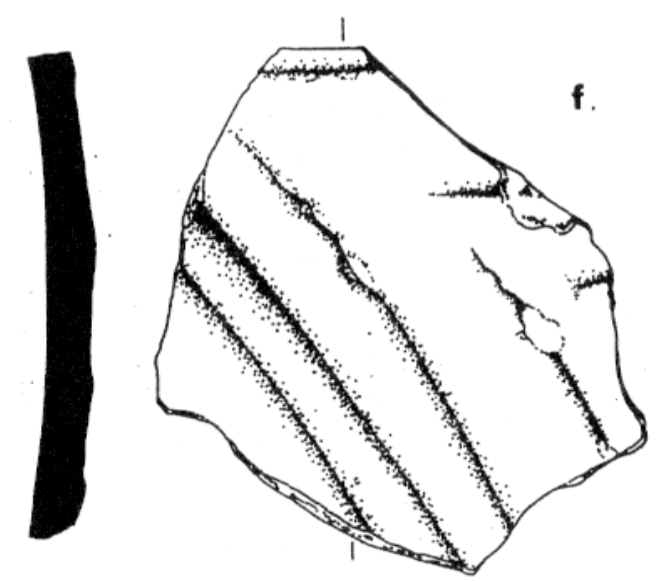

Fig. 5. - Algunos tipos cerámicos de «El Palacio de San Antonio Carupo ».

a. Tipo Carupo Rojo con negativo (Asol. GH, El. 2)

b. Tipo Chilar con negativo negro, Variedad Vertiente (Asol. GH, niv. 4)

c. Tipo Palacio pulido, Variedad Lerma (Asol. L, niv. 4)

d.e. Tipo Palacio inciso, Variedad Lerma (Asol. M, niv. 5 y M, niv. 4)

f. Tipo Hornos fino (Asol. M, niv. 5) 
una simple reutilización de una parte de la estructura con fines estrictamente funerarios parece confirmada por la ausencia de los tipos cerámicos más tardíos en el material de excavación.

\section{LA ORIGINALIDAD DE SAN ANTONIO CARUPO : ALGUNOS INDICIOS}

La organización interna del sitio y la morfología de los edificios de San Antonio Carupo manifiestan una indudable originalidad en la región : por una parte se distinguen de las de los sitios del Clásico Tardío, pero también parecen constituir un modelo bastante aislado dentro de los sitios locales contemporáneos. Antes de intentar algunas comparaciones, examinemos brevemente la situación de los grupos humanos a fines del Clásico en esta zona de la vertiente meridional del Lerma.

Al final de la fase «Lupe » (alrededor de 800 DC), nuestra sub-región estaba ocupada de manera uniforme, distribuyéndose la población en sitios de tamaños diversos ubicados generalmente sobre pendientes a proximidad de las depresiones : los «centros" comportaban una o varias zonas cívico-ceremoniales bien desarrolladas y varios sectores de habitación ; los «pueblos » tenían zonas con vocación pública más reducidas y las "aldeas » estaban compuestas únicamente de pequeños grupos de viviendas. Todos estos asentamientos, al parecer, tenían una base económica agrícola como lo indica la presencia de redes extensivas de terrazas de cultivo (B. Faugère-Kalfon, en prensa).

Alrededor de $850 \mathrm{DC}$, se notan cambios en la distribución del habitat. Aunque la agricultura es todavía, aparentemente, la actividad principal que orienta la distribución de los sitios, se observa una tendencia al agrupamiento de la población en sectores más protegidos. Sin embargo, estos cambios no afectan ni la morfología arquitectónica de las estructuras, ni tampoco la organización de éstas en los sitios.

En este marco, San Antonio Carupo aparece como un sitio estructuralmente diferente de los sitios anteriores, aun si quedan elementos que ya existían en el Clásico. De hecho, el centro ceremonial de San Antonio Carupo presenta varios elementos nuevos. Su originalidad principal yace en la ausencia de base piramidal, tipo de edificio que constituye la estructura fundamental de los recintos ceremoniales clásicos. En cambio, se encuentra una estructura cuadrada con pilares internos y pórtico externo, sin antecedente en la región. También son totalmente inéditos los recintos simétricos sin puertas, con pilares o altares rectangulares. Su cancha de juego de pelota tiene banquetas internas, áreas terminales cerradas y una orientación este-oeste, mientras que en los dos sitios verdaderamente clásicos de la zona los terrenos son abiertos y orientados norte-sur. Probablemente, la disposición linear de las estructuras y el nivelamiento regular de la pendiente no obedecen simplemente a la conformación del relieve. La creación del conjunto de tres estructuras principales (estructura con pórtico/alta plataforma con escaleras/cancha de juego de pelota) representa también una característica totalmente nueva. San Antonio Carupo presenta, por fin, un sistema constructivo en lajas poco usual, aun si el uso de lajas (sin mortero), provenientes de las barrancas vecinas, puede ser más bien una adaptación técnica a la nueva morfología de los edificios. 
Ya que El Palacio de San Antonio Carupo muestra muchos elementos nuevos, debemos interrogarnos para saber si dicho sitio constituye o no un modelo representativo del Postclásico Temprano. La respuesta es negativa. San Antonio es también bastante original en comparación con los sitios de esta época. Hecho notable, la estructura de «El Palacio», con pórtico, es la única de la región y los otros sitios contemporáneos poseen generalmente bases piramidales. Como lo hemos ya mencionado, se notan varios cambios en la repartición y la composición de los sitios a principio del Postclásico : la población se concentra más bien en «pueblos", en verdaderos sitios fortificados (cf. MICH. 400 : Las Lajas), y en grandes asentamientos situados en los contrafuertes de la meseta tarasca (G. Migeon, en preparación). Por lo tanto, llegamos a la conclusión que, si San Antonio Carupo constituye un sitio particular en el panorama del Postclásico Temprano local, su edificación coincide con cambios generales en la región.

\section{ALGUNAS HIPOTESIS INTERPRETATIVAS}

El Palacio de San Antonio Carupo aparece entonces como un sitio original que no se puede comparar con los demás sitios de la región de la misma época ; por otra parte, crea un modelo que rompe con las tradiciones arquitectónicas del Clásico. Su construcción se da en un contexto de transformación de la sociedad local, que se manifiesta en la evolución de la repartición del habitat y en la aparición de sitios defensivos. La arquitectura de San Antonio Carupo tal vez nos puede ayudar a entender estos cambios a través de un estudio de la evolución funcional de los sitios y de su interpretación a nivel macroregional.

\section{ARquitectura e ideología}

El aspecto más interesante del centro ceremonial del sitio queda en el grupo de las tres estructuras principales, grupo que se encuentra bastante aislado de los demás : la estructura cuadrangular con pórtico, la alta plataforma, que puede haber soportado super-estructuras de materiales perecederos, y la cancha de juego de pelota estaban estrechamente asociadas y comunicaban por un sistema de escaleras. Entre estas construcciones, la estructura cuadrangular y la cancha de juego de pelota se pueden interpretar en términos ideológicos.

La falta de excavaciones en la parte interna de "El Palacio ", cuya morfología no es, en sí, muy explícita, no permite atribuír una función clara a la estructura. Su ubicación y sus dimensiones indican, sin embargo, que tuvo un carácter ceremonial. Para tratar de entender su función, es necesario buscar otras estructuras similares ya excavadas. Hasta la fecha, no se han encontrado en ningún sitio de Michoacán o de Guanajuato, hecho que debemos tomar con prudencia, dada la escasez de los planos publicados $y$, por consecuencia, de las fuentes comparativas. El sitio de Nogales, Guanajuato, que se ha comparado con San Antonio (G. Zepeda García, 1988) posee, en realidad, pocos rasgos comunes con 
ćl, a excepción de la técnica constructiva : entre las estructuras principales de Nogales aparecen varias bases piramidales dispuestas sobre plazas abiertas y grandes cisternas. En cambio, en la región del río Turbio, Jalisco, podría existir una arquitectura con pórticos y recintos con altares, en particular en el sitio de «El Cuarenta " (C. Czitröm, 1985). Pero el "palacio" de San Antonio se puede más precisamente comparar con las estructuras cuadradas con columnas internas y pórticos externos de Zacatecas y de sitios pertenecientes a la cultura Chalchihuites (J.C. Kelley, 1989 ; M.A. Hers, 1989), siendo la sala de las columnas de "La Quemada » la más famosa de ellas (ver Figura 6). El «palacio» de San Antonio Carupo tiene, sin embargo, elementos distintivos : las columnas han sido cambiadas por pilares rectangulares y la columnata interna se reduce a cinco pilares agrupados alrededor de un pequeño patio ; en este sentido, representa tal vez más un "símbolo" de sala de las columnas que una copia exacta. Se sabe ahora que estos tipos de estructuras albergaban cráneos y huesos humanos, emblema guerrero y sacrificial, testigo de culturas que desarrollaron una fuerte mística militarista y que practicaban la "guerra florida" (M.A.Hers, 1989).

En este contexto, la asociación directa de tal estructura con la cancha de juego de pelota no parece ser fortuita. Es sabido que, en otras partes de Mesoamérica y por lo menos en una época tardía, los juegos de pelota se encontraban de manera privilegiada en las comunidades fortificadas o localizadas sobre fronteras, donde actos de conquista o de defensa militar determinaban la existencia misma del sitio (F.W. Fox, en prensa ; S.A. Kowalewski et alii, en prensa). Servían para solucionar conflictos políticos y territoriales y tenían entonces un carácter diplomático, político y guerrero (B. Braniff, 1988). El juego mismo habría reforzado la ideología de la élite y la noción de pertenencia a un grupo, y constituido « un cuadro ritual para sevicios corporales y ejecuciones " (S.A. Kowalewski et alii, en prensa). Si examinamos ahora la morfología de la cancha de San Antonio Carupo, constatamos que presenta una combinación de detalles poco común, tanto en Mesoamérica como en las zonas marginales del Occidente o del norte de México : es cerrada, en forma de I con pequeñas áreas terminales (tipo VIII en E. Taladoire, 1981), pero, a la vez, posee una banqueta y un muro vertical (tipo III en el mismo autor). Representa entonces una mezcla de dos tipos bien definidos, tipos que tendrían orígenes opuestos : mientras que el tipo cerrado sería originario de Mesoamérica nuclear, el tipo III viene evidentemente de Occidente (B. Braniff, $1988: 68$ ). Sin que este elemento aislado pueda tener valor de demostración, recordaremos que son perceptibles en la época Clásica Tardía local (fases «Lupe " : 600-850 DC y « La Joya " : 800-850 DC) influencias tanto del altiplano central, y en particular de Teotihuacán, como del Occidente de México (D. Michelet et alii, 1989; B. Faugère Kalfon, en preparación).

La estrecha asociación de la cancha de juego de pelota y de la estructura con pórtico externo nos ubica sin dudas en un contexto militarista y sacrificial. Este aspecto se ve todavía reforzado si se analiza la serie de estructuras ubicadas al norte del grupo principal. Estos cuartos y/o recintos regulares, algunos de los cuales simétricos, no tienen puertas y el acceso al interior de ellos se debía hacer por los techos o la parte alta de los muros : adquieren así un aspecto defensivo que corresponde al carácter militar del sitio, en el cual era necesario disponer de lugares 
a,

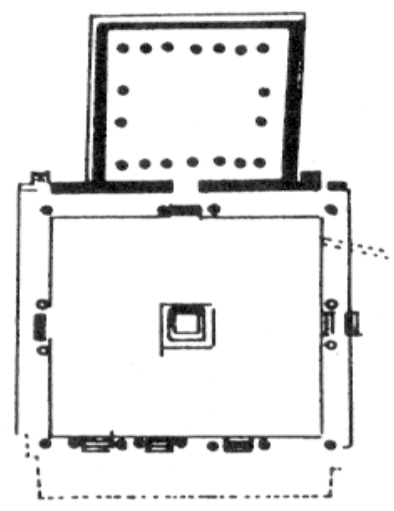

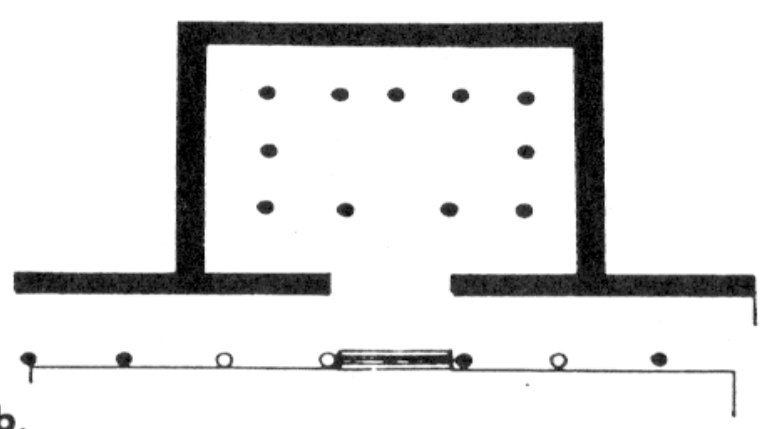

b.

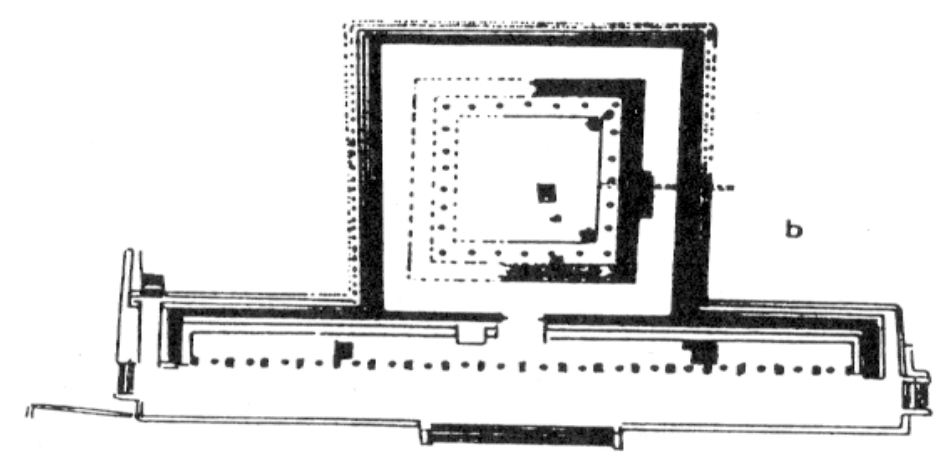

c.

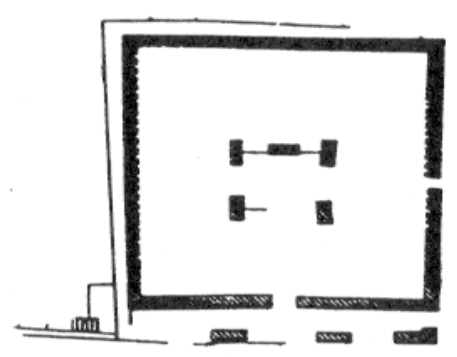

d.

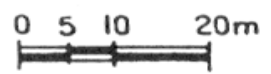

Fig. 6. - Ejemplos de salas de las columnas.

a. Alta Vista, Fase Canutillo (Kelley, 1976, p. 25, en Hers, 1989)

b. La Quemada (Guillemain Tarayre, 1867, Fig. 10, en Hers 1989)

c. «El Mercado» de Chichen Itza (Ruppert, 1943, Fig. 1, en Hers 1989)

d. El Palacio de San Antonio Carupo, Fase Palacio (900-1200 DC) 
protegidos para conservar granos y/o armas. Las mismas estructuras se encuentran, por lo demás, en sitios fortificados de las regiones norteñas, como, por ejemplo, en algunos sitios de la cultura San Gabriel de Zacatecas-Durango (R.H. Brooks, 1978).

Las estructuras arquitectónicas del centro ceremonial de San Antonio Carupo parecen fuertemente ligadas a una ideología militarista. Esta característica se podría quizás relacionar con ciertos fenómenos que se desarrollaban en la misma época en zonas muy extensas del centro-norte de México.

\section{LAS MigRACIONES PROTO-TOLTECAS.}

A partir de $800 \mathrm{DC}$, se producen profundos cambios en la zona centrooccidental de México, revelados por la discontinuidad de la ocupación humana; progresivamente los sectores más septentrionales son abandonados (B. Braniff, 1989 ; C. Castañeda et alii, 1989 ; M.A. Hers, 1988 ; J.C. Kelley, 1989 ; C.D. Trombold, 1990) y los más occidentales conocen reorganizaciones con fuertes bajas demográficas (P.C. Weigand, 1989). Se piensa que una parte de estas poblaciones sedentarias belicosas emigraron hacia el sur, y en particular hacia el altiplano central, donde contribuyeron a formar la entidad tolteca. Ahora bien, el periodo comprendido entre 850 y 1200 DC corresponde, en la región ubicada al sur del Lerma, a un auge demográfico (acompañado, como ya hemos visto, de cambios en el patrón de asentamiento), a la multiplicación de sitios protegidos y a la aparición de rasgos arquitectónicos nuevos, característicos de una ideología fuertemente impregnada de militarismo. La sincronía con la cual se desarrollan estas evoluciones y las semejanzas notadas entre ciertos elementos arquitectónicos permiten formular la hipótesis de que las migraciones proto-toltecas tuvieron un impacto en nuestra región y vinieron a estimular una transformación interna de la sociedad local. Incluso, no es imposible que haya habido un real aporte de población norteña, bien que cuantitativamente limitado. En realidad no se puede hablar de una influencia directa de Tula en nuestra zona, ya que el desarrollo del sitio de San Antonio Carupo corresponde cronológicamente a la fase Corral Terminal del Altiplano, en la cual solamente se inicia el florecimiento de la ciudad epónima de los toltecas (R.H. Cobean y A.G. Mastache, 1989); en San Antonio faltan los elementos toltecas más representativos (el plomizo o las figurillas Mazapa).

Para concluir, recordemos que se observan notables cambios en la zona de la vertiente meridional del Lerma entre el final del Clásico y el principio del Postclásico Temprano. Esta evolución podría relacionarse con las migraciones proto-toltecas que afectan el centro-occidente de México en la misma época. El auge demográfico se termina en nuestra sub-región al final del Postclásico Temprano, cuando la mayor parte de la población local empieza a emigrar hacia las tierras altas de la Meseta Tarasca, donde aparecen los primeros sitios propiamente tarascos (B. Faugère-Kalfon, en preparación). Se sugiere entonces que parte de los proto-tarascos había recibido una influencia de las culturas prototoltecas del centro-norte de México, o que los dos grupos tenían quizás razgos étnico-culturales comunes. Esta hipótesis es particularmente atractiva si se consi- 
dera la génesis del pueblo tarasco tal como la relata la Relación de Michoacán : la fracción de guerreros "chichimecas" que empieza a conquistar el poder en Michoacán en el siglo XIII podría provenir, como ya lo pensaba Jiménez Moreno en los años 40, de las regiones inmediatamente al norte (entre Guanajuato, Jalisco y Zacatecas). El uso del término "chichimecas", en este contexto, serviría para designar poblaciones sedentarias y belicosas, llevando consigo la «tradición" norteña y viniendo de zonas de contacto con nómadas o sociedades de estructura más flexible que practicaban, cuando se hacía necesario, una economía mixta de cultivo y/o de caza-recolección. También, se puede pensar que los paralelismos observados entre los mitos de origen de las sociedades azteca y tarasca (ver D. Michelet, 1989) podrian derivar, en parte, de un substrato cultural proto-tolteca común. Pero faltan ciertamente muchos trabajos para elucidar a fondo fenómenos tan complejos e importantes. *

* Manuscrit reçu en mars 1991, accepté pour publication en mai 1991.

\section{NOTA}

1. Esta vertiente constituye hoy en día un medio ecológico bastante degradado donde subsiste una vegetación escasa, compuesta de un matorral sub-tropical y de algunos encinos. Esta degradación se debe en gran parte a la elaboración de carbón, actividad particularmente común en esta región de Michoacán al final del siglo XIX y durante la primera mitad del siglo XX (la traducción de "corupo " según Lumholtz es "quemado en superficie ").

\section{BIBLIOGRAFIA}

Arnauld, M.-C., Michelet, D. - en prensa - « Les migrations postclassiques au Michoacan et au Guatemala : problèmes et perspectives ». Volume à la mémoire de $N$. Percheron. CEMCA/PUM, Toulouse.

Braniff, B., 1988. - «A propósito del ulama en el norte de México ». Arqueología, 3, Dirección de Monumentos Prehispánicos, México, INAH, 57-94.

-, 1989. - «Oscilación de la frontera norte mesoamericana : un nuevo ensayo ». Arqueología (Segunda Epoca), 1, México, INAH, 99-114.

Brooks, R. H., 1978. - «A Loma San Gabriel/Chalchihuites cultural manifestation in the Rio Ramos region, Durango, México ". In : Across the Chichimec sea. Papers in honor of J.C. Kelley. C. L. Riley y B. C. Hedrick eds. Carbondale, Southern Illinois University Press, 83-126.

Brown, R. B., 1985. - «A synopsis of the archaeology of the central portion of the northern frontier of Mesoamerica ". In : The Archaeology of West and Northwest Mesoamerica. M. S. Foster y P. C. Weigand eds. Boulder, Westview Press, 219-236.

Carrasco, R., 1982. - «Informe de los trabajos... en San Antonio Carupo ". Archivo de Monumentos Prehispánicos, INAH. 
Castañeda, C., Crespo, A. M., Flores, L. M., Contreras, J. A., Saint-Charles, J. C., 1988. - «Interpretación de la historia del asentamiento en Guanajuato ». Primera Reunión sobre las Sociedades Prehispánicas en el Centro-Occidente de México. Memoria. Centro Regional de Querétaro, Cuaderno de Trabajo 1, México, INAH, 321-357.

Castañeda, C., Cervantes, B., Crespo, A. M., Flores, L. M., 1989. - « Poblamiento prehispánico en el centro-norte de la frontera mesoamericana ». Antropologia, n. 28, Boletín Oficial del INAH, Nueva Epoca, México, INAH, 34-43.

Cobean, R. H., Mastache, A. G., 1989. - « The Coyotlatelco Culture and the Origins of the Toltec State" In : Mesoamerica after the Decline of Teotihuacan. AD. 700-900. J. C. Berlo y R. A. Diehl eds, Washington, Dumbarton Oaks, 49-67.

Czitröm, C., 1985. - « Asentamientos y cerámica de los Altos de Jalisco ». Comunicación oral presentada en el Congreso "Homenaje a J. C. Kelley", Zacatecas.

Faugère-Kalfon, B. - en prensa - « La société du Classique tardif dans le centre-nord du Michoacán, Mexique ». Trace.

En preparación. - « Entre Zacapu y río Lerma : culturas en una frontera ». Cuadernos de Estudios Michoacanos, México, CEMCA.

En preparación. - «Las representaciones rupestres del centro-norte de Michoacán ». Cuadernos de Estudios Michoacanos, México, CEMCA.

Fox, J. W. - en prensa. — «The Postclassic Highland Maya Ballgame : its spatial, sociopolitical and mythic connotations ». University of Arizona Press, V. Scarborough ed.

Hers, M. A., 1988. - «Caracterización de la cultura chalchihuites » Primera Reunión sobre las Sociedades Prehispánicas en el Centro-Occidente de México. Memoria. Centro Regional de Querétaro. Cuaderno de Trabajo 1. México. INAH, 23-39.

-, 1989. - Los toltecas en tierras chichimecas. México, UNAM.

JIMÉNEZ, MoRENO, W., 1943. - « Relaciones etnológicas entre Mesoamérica y el sureste de Estados Unidos ". El Norte de México y el Sur de Estados Unidos, IIIa Mesa Redonda, México, SMA. 17-48.

-, 1948. - «Historia antigua de la zona tarasca ». El Occidente de México, IVa Mesa Redonda, México, SMA, 146-157.

KeLley J. C., 1989. - "The early post-classic in northern Zacatecas and Durango. IX to XII centuries ». In : Mesoamérica y Norte de México - Siglo IX-XII. Seminario de Arqueología "W. Jiménez Moreno », F. Sodi Miranda coord. México. INAH. Tomo II, 487-520.

KIRChHOFF, P., 1943. - «Los recolectores-cazadores del norte de México ». El Norte de México y el Sur de Estados Unidos. IIIa Mesa Redonda, México, SMA. 133-143.

—, 1956. - «La Relación de Michoacán como fuente para la Historia de la sociedad y cultura tarascas ». Estudio preliminar de la transcripción de la Relación de las ceremonias y ritos y población y gobierno de los indios de la provincia de Michoacán (1541). Madrid, Ed. Aguilar.

Kowalewski, S. A., Feinman, G., Finsten, L., Blanton, R. E. - en prensa «Prehispanic ballgames from the valley of Oaxaca, Mexico». University of Arizona Press, V. Scarborough ed.

Lumholtz, C., 1981. - El México desconocido. Colección Clásicos de Antropología, num. 11 , México, INI.

Michelet, D., 1989. - « Histoire, mythe et apologue : notes de lecture sur la seconde partie de la Relación (...) de Michoacán ". In : Enquêtes sur l'Amérique Moyenne. Mélanges 
offerts à Guy Stresser Péan, D. Michelet coord., México, INAH/Consejo Nacional para la Cultura y las Artes/CEMCA. 105-113.

En preparación. - «Elementos para una secuencia cerámica del centro-norte de Michoacán ». Cuadernos de Estudios Michoacanos, México, CEMCA.

Michelet, D., Arnaud, M. C., Fauvet-Berthelot, M. F., 1989. - « El Protecto del CEMCA en Michoacán. Etapa I : un balance ». Trace 16, México, CEMCA, 70-87.

Migeon, G., 1991. - L'habitat postclassique dans la région de Zacapu, Michoacan. Tesis de Doctorado, Universidad de Paris I.

Piña Chan, R., OI, K., 1982. - Exploraciones arqueológicas en Tingambato, Michoacán. México, INAH.

Rzedowski, J., Calderón DE Rzedowski, G., 1987. - « El bosque tropical caducifolio en la región mexicana del Bajío ». Trace, 12, México, CEMCA. 12-21.

TAladoIRE, E., 1981. - Les terrains de jeu de balle (Mésoamérique et sud-ouest des Etats-Unis). Col. Etudes mésoaméricaines II/4, Mexico, MAEFM.

-, 1989. - «Las canchas de juego de pelota de Michoacán (CEMCA : Proyecto Michoacán) ». Trace, 16, México, CEMCA. 88-100.

Trombold, C. D., 1990. - «A reconsideration of chronology for the La Quemada portion of the northern mesoamerican frontier ». American Antiquity, Vol. 55/2, 306-324.

WeIGand, P. C., 1989. - «Discontinuity : the collapse of the Teuchitlan tradition and the Early Postclassic cultures of Western Mesoamerica ". In : Mesoamerica y Norte de México - Siglo IX-XII. Seminario de Arqueología "W. Jiménez Moreno". F. Sodi Miranda coord. México. INAH. Tomo I, 215-222.

ZEPEDA GARCiA, G., 1988. - « Nogales : fortaleza tarasca en el Estado de Guanajuato ». Primera Reunión sobre las Sociedades Prehispánicas en el Centro-Occidente de México. Memoria. Centro Regional de Querétaro, Cuaderno de Trabajo 1, México, INAH. 299-307. 